\title{
Perfil del comportamiento sexual en adolescentes mexicanos de 12 a 19 años de edad. Resultados de la ENSA 2000
}

\author{
Carlos González-Garza, MC, ${ }^{(1)}$ Rosalba Rojas-Martínez, MC, ${ }^{(2)}$ María I Hernández-Serrato, Lic, ${ }^{(2)}$ \\ Gustavo 0 laiz-Fernández, $M$ en $C$. ${ }^{(2)}$
}

\begin{abstract}
González-Garza C, Rojas-Martínez R, Hernández-Serrato MI, Olaiz-Fernández G. Perfil del comportamiento sexual en adolescentes mexicanos de 12 a 19 años de edad. Resultados de la ENSA 2000. Salud Publica Mex 2005;47:209-218.
\end{abstract}

\section{Resumen}

Objetivo. D escribir las características del comportamiento sexual de los adolescentes mexicanos, su grado de conocimiento so bre anticoncepción, las variables que se relacionan con la utilización de anticonceptivos en la primera relación sexual y las que se asocian con el embarazo. Material y métodos Se analizó la base de datos de adolescentes de la Encuesta N acional de Salud 2000, realizada en septiembre de 1999 a marzo de 2000, la cual tuvo un diseño complejo al ser probabilístico, polietápico, estratificado y por conglomerados. La muestra incluyó a adolescentes de entre 12 a 19 años de edad ( $n=15241$ ). Se hizo un análisis descriptivo, se aplicó la prueba ji cuadrada (W ald) para evaluar diferencias de proporciones, y se construyeron dos modelos de regresión logística para obtener razones de momios. Resultados El $69.2 \%$ de los adolescentes refirió conocer al menos un método de control de la fecundidad. El 16.4\% mencionó haber tenido relaciones sexuales, y los hombres iniciaron la actividad sexual antes que las mujeres; es importante mencionar que tan sólo 37\% de los adolescentes usaron algún método anticonceptivo en la primera relación sexual. El análisis de regresión logística mostró que los adolescentes del sexo masculino, los de mayor escolaridad, quienes poseían información de algún método anticonceptivo y aquellos que inician esta actividad a una edad mayor tuvieron más probabilidad de usar anticonceptivos en su primera relación sexual. El $55.7 \%$ de las mujeres que mencionaron haber iniciado actividad sexual han estado embarazadas. El embarazo en las adolescentes se asoció significativamente con un nivel bajo de escolaridad, con iniciar su vida sexual a
González-Garza C, Rojas-Martínez R, Hernández-Serrato MI, Olaiz-Fernández G. Profile of sexual behavior in 12 to 19 year-old Mexican adolescents. Results of ENSA 2000. Salud Publica Mex 2005;47:209-218.

\section{A bstract}

Objective To describe traits associated with sexual behaviors in Mexican adolescents, their knowledge about contraception, and factors associated with pregnancy and utilization of contraceptives during first sexual intercourse. Material and Methods. Data from the Mexican $\mathrm{N}$ ational Health Survey 2000 (EN SA 2000) were analyzed.This study, conducted between September 1999 and March 2000, was a complex survey with a probabilistic, stratified, and cluster sampling design. The sample population included 15241 adolescents 12 to 19 years of age. Data analysis included descriptive statistics and a chi-squared test for differences of proportions; also, logistic regression was used to obtain odds ratios. Results A total of $69.2 \%$ adolescents reported knowledge of at least one contraception method; $16.4 \%$ of subjects reported having had sexual intercourse. Males initiated sexual intercourse earlier than females and only $37 \%$ of all adolescents utilized contraceptives during their first sexual intercourse. Logistic regression analysis showed that being male, having a higher education, having knowledge of at least one contraception method, and having initiated sexual intercourse at an older age, were factors associated with utilization of a contraception method in their first sexual intercourse. A total of $55.7 \%$ of sexually active female adolescents had been pregnant. Pregnancy among adolescents was associated with low educational level, sexual activity at early age, and to have sometime lived in free union. Conclusions The majority of sexually active adolescents did not use contraception during their first sexual intercourse, rendering them at risk of unwanted pregnancies.

(1) University of C alifornia, San Diego. Estados Unidos de América

(2) Instituto $N$ acional de Salud Pública. México, DF, México.

Fecha de recibido: 15 de abril de 2004 - Fecha de aprobado: 30 de marzo de 2005

Solicitud de sobretiros: Lic. María I Hernández Serrato. Instituto N acional de Salud Pública. Calle Fray Pedro de Gante \# 50, colonia Sección XVI, Delegación Tlalpan, 14080 México, DF, México.

Correo electrónico: mihernandez@insp.mx 
edades tempranas y con el hecho de haber estado alguna vez unidas. Conclusiones En general, la población adolescente que ha tenido relaciones sexuales no utilizó métodos anticonceptivos durante su primera relación, lo cual incrementa la posibilidad de embarazos.

Palabras clave: conocimiento; uso de anticonceptivos; inicio de vida sexual; embarazo; adolescencia; México
Key words: awareness; contraceptive use; sexual intercourse; pregnancy; adolescence; Mexico
L a Organización Mundial de la Salud (OMS) define a la adolescencia como la segunda década de la vida, es decir, la etapa que abarca edades entre los 10 y los 19 años. ${ }^{1}$ Otros autores la definen como el periodo de transición entre la infancia y la edad adulta, etapa caracterizada por la sucesión de importantes cambios fisiológicos, psicológicos y sociales en el ser humano. ${ }^{2-4}$ Tradicionalmente, se considera a la adolescencia como una etapa relativamente exenta de problemas de salud; sin embargo, es evidente que, aun cuando la morbilidad y la mortalidad son bajas en los adolescentes, están expuestos a factores de riesgo a la salud como el sobrepeso, el tabaquismo, el alcoholismo, los accidentes, los homicidios y los suicidios, ${ }^{5,6}$ además, deben enfrentarse durante esta etapa a su sexualidad, y evitar embarazos no deseados e infecciones de transmisión sexual. ${ }^{7-9}$

La información disponible en México sobre comportamiento, actitudes y prácticas sexuales proviene de censos, encuestas sociodemográficas y epidemiológicas, principalmente.

En relación con el conocimiento de métodos anticonceptivos, se notificó que las mujeres de 15 a 19 años de edad tienen, en general, menor conocimiento sobre métodos anticonceptivos; en 1976, 79.8\% de las mujeres conocía algún método anticonceptivo; para 1992, 90.9\%, lo que ascendió a $93.4 \%$ en $1997 .{ }^{10}$ La Encuesta Gente Joven de la Fundación Mexicana para la Planeación familiar (Mexfam), aplicada en 1999 a adolescentes de 13 a 19 años de edad, indica que $93.4 \%$ de los hombres y $80.9 \%$ de las mujeres declararon tener conocimiento formal o informal de métodos anticonceptivos. ${ }^{11}$ Para el año 2000 la Encuesta Nacional de la Juventud (ENJ2000) destaca que $81.5 \%$ de los hombres y $76 \%$ de las mujeres de entre 15 a 19 años de edad mencionaron conocer sobre métodos anticonceptivos. ${ }^{12}$

Con la información de las últimas dos décadas es difícil precisar el porcentaje de adolescentes que han iniciado su vida sexual. La Encuesta sobre el Comportamiento Reproductivo de los adolescentes y Jóve- nes del Area Metropolitana de la Ciudad de México (ECRAMM), realizada en México en 1988, señala que $41.9 \%$ de los hombres y $22.9 \%$ de las mujeres habían declarado tener relaciones sexuales a una edad promedio de 17 años en los hombres, y a los 16 años en las mujeres. En esta encuesta sobresale también que sólo $33.8 \%$ de los jóvenes con actividad sexual había utilizado algún método anticonceptivo en su primera relación. ${ }^{13}$

En 1999 la encuesta realizada por la Mexfam en jóvenes de entre 13 a 19 años de edad resalta que $22.3 \%$ de los hombres y $10.3 \%$ de las mujeres ya habían tenido relaciones sexuales, y que la edad promedio de la primera relación fue a los 15 años; no se observó diferencia entre hombres y mujeres. Además, $44 \%$ de los adolescentes sexualmente activos declararon haber empleado algún método en su primera relación. ${ }^{11} \mathrm{La}$ ENJ2000 señala que $55.3 \%$ de jóvenes de 15 a 29 años de edad tienen actividad sexual; de los jóvenes que tuvieron relaciones sexuales $67.7 \%$ declaró que la edad de inicio fue entre los 15 y los 19 años. ${ }^{12}$

El embarazo en la adolescencia se ha considerado como un problema relevante, a pesar de que las tasas específicas de fecundidad en mujeres de entre 15 a 19 años de edad han disminuido en los últimos años, ${ }^{14} \mathrm{en}$ 2000 ocurrieron más de 500 mil embarazos en adolescentes menores de 19 años, y de éstos, alrededor de 366000 llegaron a término, lo que representó $17 \%$ del total de nacimientos del país. ${ }^{15}$ En circunstancias de pobreza y desnutrición, ${ }^{14}$ el embarazo a edades tempranas puede representar un riesgo para la salud de la adolescente y el recién nacido. ${ }^{16-18}$ Se sabe también que el embarazo en edades tempranas puede atribuirse a la edad, el estado civil, el nivel de escolaridad de la mujer, ${ }^{19}$ y las condiciones socioeconómicas y culturales de la población, entre otros. ${ }^{14}$

Existe una gran discrepancia en los resultados de las encuestas para investigar el comportamiento sexual en este sector de la población; no son comparables, principalmente por la inclusión de diferentes interva- 
los de edad, por la variación en la conceptualización de las variables, por los instrumentos para recolectar la información y por el tipo de muestreo.

La Encuesta Nacional de Salud 2000 (ENSA 2000) proporciona información reciente del comportamiento sexual de los adolescentes en México y permite estimaciones a escala nacional y regional. En este trabajo nos propusimos analizar el conocimiento sobre anticoncepción, el inicio de la vida sexual, los factores que intervienen en el uso de anticonceptivos en la primera relación sexual, así como los que se asocian con el antecedente de embarazo entre los adolescentes mexicanos.

\section{Material y métodos}

La ENSA 2000 es de tipo probabilístico, polietápico, estratificado y de conglomerados; tiene representatividad nacional y estatal, y el tamaño de la muestra fue de 45726 viviendas. La metodología, el diseño de la encuesta, el cálculo del tamaño de muestra y la estimación de ponderadores han quedado descritos en otra publicación. ${ }^{20}$ Los padres de los adolescentes que participaron en la encuesta firmaron una carta de consentimiento informado, de acuerdo a los "Principios éticos para las investigaciones médicas en seres humanos", de la Declaración de Helsinki.

Para el desarrollo de este artículo se manejaron dos bases de datos de la ENSA 2000; las variables corresponden a la sección de salud reproductiva del cuestionario de adolescentes, los datos demográficos y del ingreso familiar se obtuvieron del cuestionario del hogar. Se incluyó a adolescentes de 12 a 19 años de edad con cuestionario completo, fueron excluidos los adolescentes que tuvieron la primera relación sexual entre los 2 y los 7 años de edad (0.2). De esa manera, la población estuvo integrada por 15241 que al aplicar los factores de ponderación representan a 16491476 adolescentes: $48.6 \%$ hombres y $51.4 \%$ mujeres.

La escala original de respuestas de algunas variables se recodificaron a variables dicotómicas para efectuar el análisis estadístico; construimos la variable inicio de vida sexual, en la cual consideramos que sí habían iniciado la actividad sexual aquellos adolescentes que manifestaron haber tenido su primera relación sexual entre los 8 y los 19 años de edad. En esta categoría incluimos también a mujeres que dijeron desconocer la edad de inicio de la vida sexual y en quienes existía el antecedente de embarazo. Del mismo modo, se consideró que sí usaron algún método anticonceptivo aquellos casos en que los adolescentes recurrieron al uso de un método en su primera relación. Para el antecedente de embarazo se consideraron a las adolescentes que informaron haber estado embarazadas alguna vez. El ingreso del hogar se estableció a partir del salario mínimo mensual que reciben todos sus integrantes mayores de 12 años de edad, de acuerdo al salario mínimo vigente por municipio en el año 2000; se categorizó en dos grupos, los que tienen ingresos menores a tres salarios y aquellos que perciben tres o más salarios mensuales. Sobre el lugar de residencia, consideramos una localidad urbana cuando el número de habitantes fue igual o mayor a 15000 , y localidad rural, con un número de entre 1 a 14999 habitantes. La variable estado civil se recategorizó en dos grupos: en el primero clasificamos a los(as) solteros(as), y en el segundo se incluyó a quienes declararon estar casados(as), los que viven en unión libre, divorciados(as), separados(as) y viudos(as) al momento de la entrevista.

Se hizo un análisis descriptivo para obtener frecuencias simples de cada una de las variables de estudio con el fin de detectar valores fuera de rango y conocer la distribución de cada variable. Para poder expandir los resultados a la población adolescente de entre 12 a 19 años de edad, en el análisis se tomó en cuenta el peso relativo asignado a cada una de las observaciones de acuerdo con el esquema de muestreo utilizado. En el análisis bivariado se efectuaron pruebas de Ji cuadrada (Wald) para evaluar diferencias de proporciones entre el conocimiento de algún método anticonceptivo, inicio de vida sexual y antecedente de embarazo, según categorías de determinadas características. Finalmente, en el análisis multivariado se hizo regresión logística con la que analizamos el uso de métodos anticonceptivos en la primera relación sexual y el antecedente de embarazo como variables dependientes; se consideraron como variables independientes la edad, el sexo, la escolaridad, el estado civil, la derechohabiencia, el lugar de residencia, el conocimiento sobre métodos anticonceptivos y la edad en la primera relación sexual. Para el análisis estadístico se utilizaron los paquetes estadísticos STATA, SAS y SUDAAN.

\section{Resultados}

Respecto al conocimiento que los adolescentes tienen sobre métodos anticonceptivos, $69.2 \%$ mencionó conocer al menos un método anticonceptivo; en el cuadro I se advierte un incremento en el porcentaje conforme aumenta la edad, observamos diferencias significativas: $54.2 \%$ de los de 12 a 14 años de edad dijeron conocer al menos un método anticonceptivo, el mayor porcentaje estuvo en los de 18 a 19 años de edad $(p=0.0000)$. El $51.6 \%$ de los hombres de 12 a 14 años declararon conocer al menos un método anticonceptivo, 5.1 puntos 


\section{Cuadro I \\ Porcentaje de adolescentes de entre 12 a 19 años de edad Que conocen al menos un método anticonceptivo, SEgún Características seleccionadas. México, ENSA 2000}

\begin{tabular}{|c|c|c|c|c|c|c|c|}
\hline & \multirow[b]{4}{*}{ N } & \multirow[b]{4}{*}{$\mathrm{N}^{*}$} & \multicolumn{5}{|c|}{ Conocimiento de métodos anticonceptivos } \\
\hline & & & \multirow{3}{*}{$\begin{array}{l}\text { No } \\
\%\end{array}$} & \multirow{3}{*}{$\begin{array}{l}\text { Sí } \\
\%\end{array}$} & \multicolumn{3}{|c|}{ Edad (años) } \\
\hline & & & & & 12 a 14 & 15 a 17 & 18 a 19 \\
\hline Variable & & & & & $\%$ & $\%$ & $\%$ \\
\hline \multicolumn{8}{|l|}{ Sexo } \\
\hline Hombres & 6758 & 8006809 & 32.4 & 67.6 & 51.6 & 75.1 & 83.7 \\
\hline Mujeres & 8483 & 8484667 & 29.4 & $70.6^{\ddagger}$ & $56.7^{\ddagger}$ & 77.6 & 83.2 \\
\hline \multicolumn{8}{|l|}{ Escolaridad\# } \\
\hline Sin escolaridad & 110 & 149229 & 78.3 & 21.7 & 31.9 & 20.1 & 15.9 \\
\hline Primaria & 5525 & 5968320 & 56.3 & 43.7 & 40.0 & 43.8 & 60.6 \\
\hline Secundaria o técnica & 6833 & 7331078 & 19.8 & 80.2 & 75.2 & 82.4 & 84.2 \\
\hline Preparatoria y más & 2607 & 2883653 & 3.8 & $96.2^{\ddagger}$ & $\S$ & $95.7^{\ddagger}$ & $96.8^{\ddagger}$ \\
\hline \multicolumn{8}{|l|}{ Estado civil ${ }^{\S}$} \\
\hline Soltero (a) & 13978 & 15325492 & 31.8 & 68.2 & 54.2 & 76.0 & 82.9 \\
\hline Alguna vez unidos (as) & 1200 & 1089813 & 16.3 & $83.7^{\ddagger}$ & 69.6 & 81.2 & 85.7 \\
\hline \multicolumn{8}{|l|}{ Acceso a servicio de salud } \\
\hline Sí & 5980 & 5947932 & 19.7 & 80.3 & $66.3^{\ddagger}$ & $87.6^{\ddagger}$ & $91.4^{\ddagger}$ \\
\hline No & 9261 & 10543544 & 37.0 & $63.0 \mathrm{a}$ & 47.9 & 70.3 & 79.0 \\
\hline \multicolumn{8}{|l|}{ Ingreso en el hogar } \\
\hline$>=3$ salarios mínimos & 6882 & 7702807 & 24.8 & $75.2^{\ddagger}$ & $60.7^{\ddagger}$ & $80.9^{\ddagger}$ & $87.01^{\ddagger}$ \\
\hline$=3$ salarios mínimos & 7846 & 8229522 & 36.0 & 64.0 & 49.2 & 72.7 & 80.7 \\
\hline \multicolumn{8}{|l|}{ Lugar de residencia } \\
\hline Urbana & 8043 & 9369477 & 20.6 & $79.3^{\ddagger}$ & $64.8^{\ddagger}$ & $85.7^{\ddagger}$ & $90.5^{\ddagger}$ \\
\hline Rural & 7198 & 7121999 & 44.2 & 55.8 & 42.8 & 63.6 & 70.9 \\
\hline Total & 15241 & 16491476 & 30.8 & 69.2 & 54.2 & 76.3 & 83.4 \\
\hline * Muestra ponderada & \multicolumn{2}{|c|}{ \# Sin información: 1\% } & & & & & \\
\hline$\neq P<0.001$ & \multicolumn{2}{|c|}{ \& Sin información: 0.4\% } & & & & & \\
\hline \$ No aplica & \multicolumn{2}{|c|}{ F Sin información: 3.3\% } & & & & & \\
\hline
\end{tabular}

porcentuales menor al de las mujeres $(p=0.0129)$, mientras que no se observaron diferencias significativas en los de 15 a 19 años de edad en uno y otro sexo. También existieron diferencias significativas en el conocimiento de acuerdo con la escolaridad, $31.9 \%$ de los adolescentes sin escolaridad en el grupo de edad de 12 a 14 años mencionaron conocer algún método anticonceptivo y se apreció un aumento en la proporción de conocimiento conforme se incrementó la escolaridad; de la misma manera, se observó una tendencia significativa $(p=0.000)$ entre mayor escolaridad mayor cono- cimiento de métodos en los jóvenes de los 15 a los 19 años de edad.

Por otra parte, aumentó la proporción de los adolescentes que refirieron conocer al menos un método anticonceptivo en aquellos que tienen acceso a algún servicio médico $(p=0.0000)$, en los jóvenes con ingreso en el hogar mayor a tres salarios mínimos $(p=0.0000)$, $\mathrm{y}$ en los que viven en áreas urbanas $(p=0.0000)$.

En relación con el inicio de vida sexual se encontró que $16.4 \%$ la habían iniciado; para caracterizar mejor el comportamiento sexual entre los adolescen- 
tes se analizó por grupos de edad. En el cuadro II hay un aumento en el porcentaje de inicio de vida sexual, casi sin actividad sexual en los adolescentes de los 12 a 14 años de edad, $16.7 \%$ en los de 15 a 17, y $42.9 \%$ en los de 18 y 19 años $(p=0.0000)$. Se destaca un mayor porcentaje de inicio de actividad sexual en los hombres de 18 a 19 años ( $p=0.0028)$. Respecto a la escolaridad se observa el mayor porcentaje de inicio de vida sexual en adolescentes de 15 a 19 años sin escolaridad, y después hay una disminución en los porcentajes de acuerdo al aumento de la escolaridad $(p=0.0000)$. Asimismo, sobresale una mayor actividad sexual en aquellos de entre 15 a 17 años de edad que viven en localidades urbanas comparados con los que viven en localidades rurales (19.2 y 13.6\%, respectivamente; $p=0.0001$ ).

Del total de adolescentes que tuvieron relaciones sexuales, 37\% dijo haber utilizado algún método anticonceptivo durante su primera relación; el porcentaje de utilización de algún método anticonceptivo en la primera relación sexual es más alto en los hombres que en las mujeres $(p=0.000)$. En el cuadro III se encuentran las variables que favorecen la utilización de anticonceptivos durante la primera relación; se observa que los hombres usan 4.09 (IC 95\% 3.12 - 5.36) veces más

\section{Porcentaje de adolescentes de entre 12 a 19 años de edad con inicio de vida sexual, SEGÚN VARIABLES SELECCIONADAS. MÉXICO, ENSA 2000}

\begin{tabular}{|c|c|c|c|c|c|c|}
\hline & & & & Inicio & sexual & \\
\hline & & & & & Edad (años & \\
\hline & & & Total & 12 a 14 & 15 a 17 & 18 a 19 \\
\hline Variable & $\mathrm{N}$ & N* & $\%$ activos & $\%$ & $\%$ & $\%$ \\
\hline Sexo & & & & & & \\
\hline Hombres & 6758 & 8006809 & 16.7 & 1.1 & 16.6 & $47.0^{\ddagger}$ \\
\hline Mujeres & 8483 & 8484667 & 15.6 & 1.1 & 16.7 & 39.3 \\
\hline Escolaridad\& & & & & & & \\
\hline Sin escolaridad & 110 & 149229 & $28.6^{\ddagger}$ & $\S$ & $38.2^{\ddagger}$ & $37.6^{\ddagger}$ \\
\hline Primaria & 5525 & 5968320 & 13.3 & 1.0 & 25.5 & 55.3 \\
\hline Secundaria o técnica & 6833 & 7331078 & 16.6 & 1.3 & 15.7 & 48.2 \\
\hline Preparatoria y más & 2607 & 2883653 & 20.7 & $\#$ & 9.8 & 32.1 \\
\hline Estado civil ${ }^{\neq}$ & & & & & & \\
\hline Soltero (a) & 13978 & 15325492 & 10.5 & 0.9 & 11.4 & 30.3 \\
\hline Alguna vez unidos (as) & 1200 & 1089813 & $95.0^{\ddagger}$ & $72.3^{\ddagger}$ & $92.2^{\ddagger}$ & $97.5^{\ddagger}$ \\
\hline Acceso a servicio de salud & & & & & & \\
\hline Sí & 5980 & 5947932 & 15.7 & 0.9 & 14.5 & 42.3 \\
\hline No & 9261 & 10543544 & 16.3 & 1.3 & $18.5^{\ddagger}$ & 42.9 \\
\hline Ingreso en el hogar ${ }^{\infty}$ & & & & & & \\
\hline$>=3$ salarios mínimos & 6882 & 7702807 & $17.4^{\ddagger}$ & 1.4 & $17.2^{\ddagger}$ & 41.3 \\
\hline$<=3$ salarios mínimos & 7846 & 8229522 & 14.6 & 1.0 & 15.9 & $43.8^{\ddagger}$ \\
\hline Lugar de residencia & & & & & & \\
\hline Urbana & 8043 & 9369477 & $18.7^{\ddagger}$ & 1.1 & $19.2^{\ddagger}$ & 44.3 \\
\hline Rural & 7198 & 7121999 & 12.8 & 1.1 & 13.6 & 40.4 \\
\hline Total & 15241 & 16491476 & 16.4 & 1.1 & 16.7 & 42.9 \\
\hline $\begin{array}{l}\text { * Muestra ponderada } \\
\text { ₹ } p<0.01 \\
\S \text { Sin datos } \\
\text { \# No aplica }\end{array}$ & $\begin{array}{l}\& \text { Sin información: } 1 \% \\
\text { ₹ Sin información: } 0.4 \% \\
{ }^{\infty} \text { Sin información: } 3.3 \%\end{array}$ & & & & & \\
\hline
\end{tabular}




\section{Cuadro III \\ Factores que determinan el uso de métodos anticonceptivos* en la primera Relación sexual en ADolescentes de 12 a 19 años de edAd. México, ENSA 2000}

\begin{tabular}{|c|c|c|c|c|c|c|}
\hline Variable & N* & $N^{\ddagger}$ & Nivel de significancia & $\mathrm{RM}^{\S}$ & \multicolumn{2}{|c|}{ IC $95 \%$} \\
\hline \multicolumn{7}{|l|}{ Sexo } \\
\hline Mujer\# & 1446 & 1310020 & & 1.00 & & \\
\hline Hombre & 1088 & 1324513 & 0.0000 & 4.09 & 3.12 & 5.36 \\
\hline \multicolumn{7}{|l|}{ Escolaridad } \\
\hline $\mathrm{N}$ inguna o primariä & 818 & 822605 & & 1.00 & & \\
\hline Secundaria & 1137 & 1203108 & 0.0001 & 2.05 & 1.43 & 2.96 \\
\hline Preparatoria y más & 564 & 594349 & 0.0000 & 3.56 & 2.34 & 5.41 \\
\hline \multicolumn{7}{|l|}{ Lugar de residencia } \\
\hline Rural” & 1067 & 898357 & & 1.00 & & \\
\hline Urbana & 1467 & 1736176 & 0.0010 & 1.94 & 1.48 & 2.55 \\
\hline
\end{tabular}

Edad de la primera relación sexual

\begin{tabular}{lrrrrrr}
8 a 13 años & 234 & 225377 & 1.00 & \\
\hline 14 a 15 años & 827 & 832726 & 0.0428 & 1.76 & 1.02 & 3.16 \\
\hline 16 a 17 años & 1095 & 1171529 & 0.0111 & 2.22 & 1.20 & 4.11 \\
\hline 18 a 19 años & 378 & 404901 & 0.0009 & 3.16 & 1.60 & 6.23
\end{tabular}

Total

$2534 \quad 2634533$

* Sin información: $1.2 \%$

‡ Muestra ponderada

§ Las razones de momios ajustadas para la edad, sexo, escolaridad, lugar de residencia, y edad de la primera relación sexual; IC $95 \%$ : intervalo de confianza de $95 \%$

* Categoría de referencia

algún anticonceptivo que las mujeres y la escolaridad es un factor que influye en la utilización de anticonceptivos; quienes tienen un nivel de secundaria utilizan 2.05 (IC 95\%, 1.43-2.96) veces más anticonceptivos en la primera relación sexual, y 3.56 (IC 95\%, 2.34-5.41) veces para los de escolaridad preparatoria o mayor nivel escolar comparado con aquellos con educación primaria. Los adolescentes que viven en localidades urbanas tienen 1.94 (IC 95\%, 1.48-2.55) veces más posibilidad de usar anticonceptivos en la primera relación sexual, comparados con aquellos que viven en localidades rurales. Asimismo, la edad en la primera relación sexual es un factor que influye para el uso de algún método anticonceptivo; quienes tuvieron su primera relación entre los 18 y 19 años de edad emplean 3.16 (IC 95\%, 1.60-6.23) veces más algún método anticonceptivo, y quienes iniciaron a los 16 y 17 años tienen dos (IC 95\%, 1.20-4.11) veces más posibilidad de recurrir a algún método anticonceptivo que aquellos que tuvieron su primera relación sexual entre los 8 y 13 años de edad.

En cuanto al antecedente de embarazo en las adolescentes de entre 12 a 19 años de edad que han tenido relaciones sexuales, $55.7 \%$ han estado embarazadas alguna vez. En el cuadro IV se muestra que el porcentaje de antecedente de embarazo aumenta de acuerdo con la edad de las adolescentes ( $p=0.0001$ ): $14.8 \%$ de las jóvenes de los 12 a 14 años, $48 \%$ en las de 15 a 17 años, y $63.2 \%$ en las de 18 a 19 años de edad; asimismo, observamos un aumento del porcentaje de embarazo en las características estudiadas debido al efecto de la edad. El antecedente de embarazo según nivel de escolaridad al momento de la encuesta presenta un comportamiento inverso; a menor nivel escolar mayor porcentaje de adolescentes con antecedente de embarazo, la diferencia más significativa se presenta en el grupo de los 18 a los 19 años de edad $(p=0.0000)$. Por otra parte, al presentar los porcentajes más altos de 


\section{Cuadro IV \\ Porcentaje de antecedente de embarazo en mujeres de entre 12 y 19 años de edad con inicio DE VIDA SEXUAL,* SEgún VARIables SELeCCIONAdAs. México, ENSA 2000}

\begin{tabular}{|c|c|c|c|c|c|c|c|}
\hline & & & & & idente de & & \\
\hline & & & & & & Edad & \\
\hline & & & No & Sí & $\overline{12 \text { a } 14}$ & 15 a 17 & 18 a 19 \\
\hline Variable & $\mathrm{N} *$ & $\mathrm{~N}^{\ddagger}$ & $\%$ & $\%$ & $\%$ & $\%$ & $\%$ \\
\hline Escolaridad ${ }^{\neq}$ & & & & & & & \\
\hline Sin escolaridad & 34 & 35125 & 32.7 & $67.3^{\S}$ & \# & 43.4 & $95.4^{\S}$ \\
\hline Primaria & 549 & 486609 & 37.6 & 62.4 & 23.9 & 50.1 & 76.6 \\
\hline Secundaria o técnica & 637 & 573023 & 44.8 & 55.2 & 4.2 & 45.6 & 65.8 \\
\hline Preparatoria y más & 214 & 198338 & 62.7 & 37.3 & $\&$ & 51.7 & 33.0 \\
\hline Estado civil ${ }^{\infty}$ & & & & & & & \\
\hline Soltera & 448 & 501577 & 66.9 & 33.1 & 6.0 & 28.5 & 38.9 \\
\hline Alguna vez unidas & 993 & 795779 & 30.6 & $69.6^{\S}$ & 28.7 & $59.9 a$ & $78.1^{\S}$ \\
\hline Ingreso en el hogar ${ }^{\varnothing}$ & & & & & & & \\
\hline$\geq=3$ salarios mínimos & 572 & 577461 & 52.1 & 47.9 & 5.0 & 39.8 & 55.6 \\
\hline$<3$ salarios mínimos & 819 & 666587 & 33.1 & $66.9^{\S}$ & 27.1 & $55.4 a$ & 68.9 \\
\hline Conocimiento de métodos & & & & & & & \\
\hline Sí & 1245 & 1089118 & 53.0 & 47.0 & 18.7 & $51.2 a$ & 62.7 \\
\hline No & 198 & 209358 & 42.8 & 57.2 & 10.7 & 34.0 & 67.4 \\
\hline
\end{tabular}

Edad de la primera relación sexual

\begin{tabular}{lrrrrrrr}
8 a 13 años & 98 & 82216 & 55.9 & $44.1^{\S}$ & 11.8 & 49.3 & $83.6^{\S}$ \\
\hline 14 a 15 años & 472 & 410802 & 39.0 & 61.1 & 19.7 & 54.4 & 76.0 \\
\hline 16 a 17 años & 642 & 602600 & 39.8 & 60.2 & $\&$ & 41.1 & 73.2 \\
\hline 18 a 19 años & 231 & 202850 & 65.9 & 34.1 & $\&$ & $\&$ & 34.2
\end{tabular}

Uso de anticonceptivos en la primera relación ${ }^{\diamond}$

\begin{tabular}{|c|c|c|c|c|c|c|c|}
\hline Sí & 1149 & 249255 & 55.9 & 44.1 & 16.2 & 34.0 & 51.8 \\
\hline No & 276 & 1034651 & 41.8 & $58.2^{\S}$ & 14.1 & 50.8 & $65.9^{\S}$ \\
\hline Total & 1443 & 1298476 & 44.3 & 55.7 & 14.8 & 48.0 & 63.2 \\
\hline $\begin{array}{l}\text { * Sin información en } 1.5 \% \text { de los casos } \\
\text { ₹ Muestra ponderada } \\
\text { \& p } 0.001 \\
\text { \# sin datos } \\
\& \text { po anlica }\end{array}$ & & \multicolumn{6}{|c|}{$\begin{array}{l}\text { * Sin información: } 0.6 \% \\
{ }^{\infty} \text { Sin información: } 0.1 \% \\
{ }^{\circ} \text { Sin información: } 3.6 \% \\
\diamond \text { Sin información: } 1.2 \%\end{array}$} \\
\hline
\end{tabular}

antecedente de embarazo se advierten diferencias estadísticamente significativas entre las adolescentes mayores de 14 años con ingreso familiar menor a tres salarios mínimos, comparadas con las de ingreso familiar igual o mayor que tres, y en las que están o estuvieron unidas alguna vez, comparadas con las solteras. Se observa que el inicio de relaciones sexuales antes de los 16 años de edad aumenta el porcentaje de adolescentes que han estado embarazadas alguna vez, siendo más significativo en las mujeres de 18 a 19 años $(p=0.0000)$. Acerca del uso de métodos en la primera relación sexual hay diferencias, la mayor proporción de antecedente de embarazo fue en las mujeres de 18 a 19 años de edad que no usaron ningún tipo de protección ( $p=0.0287$ ).

En el cuadro V se hallan las variables que en el modelo de regresión logística estuvieron relacionadas con el antecedente de embarazo; en cuanto a la escola- 


\section{Cuadro V \\ Factores asociados con el embarazo en las adolescentes de entre 12 y 19 años de edad. MÉxICO, ENSA 2000}

\begin{tabular}{|c|c|c|c|c|c|c|}
\hline Variable & $\mathrm{N}$ & $\mathrm{N}^{*}$ & Nivel de significancia & $\mathrm{RM}^{\ddagger}$ & \multicolumn{2}{|c|}{ IC $95 \%$} \\
\hline \multicolumn{7}{|l|}{ Escolaridad } \\
\hline Preparatoria y más§ & 214 & 198338 & & & & \\
\hline Secundaria o técnica & 637 & 573023 & 0.012 & 1.96 & 1.16 & 3.33 \\
\hline Primaria & 583 & 521734 & 0.009 & 2.22 & 1.22 & 4.02 \\
\hline \multicolumn{7}{|c|}{ Edad en la primera relación sexual } \\
\hline 18 a 19 años $s^{\S}$ & 231 & 202850 & & 1.00 & & \\
\hline 16 a 17 años & 642 & 602600 & 0.000 & 5.96 & 3.61 & 9.84 \\
\hline 14 a 15 años & 472 & 410802 & 0.000 & 13.84 & 6.55 & 29.13 \\
\hline 8 a 13 años & 98 & 82216 & 0.000 & 12.29 & 4.40 & 34.34 \\
\hline \multicolumn{7}{|l|}{ Estado civil ${ }^{\S}$} \\
\hline Solteras & 448 & 501577 & & 1.00 & & \\
\hline Alguna vez unidas & 993 & 795779 & 0.000 & 4.52 & 2.85 & 7.18 \\
\hline Total & 1443 & 1298476 & & & & \\
\hline \multicolumn{7}{|l|}{ * Muestra ponderada } \\
\hline $\begin{array}{l}\ddagger \text { Las razones de momios } \\
\S \text { Categoría de referencia }\end{array}$ & lad, esco & dad de la pr & relación sexual y est & I:IC 95 & & $95 \%$ \\
\hline
\end{tabular}

ridad al momento de la encuesta, se observó que cuando fue primaria las adolescentes tuvieron mayor riesgo de embarazo (RM 2.22, IC 95\% 1.22-4.02) en comparación con aquellas de mayor escolaridad. De modo similar, el inicio temprano de la vida sexual es un factor que influye de manera importante sobre el antecedente de embarazo; las jóvenes que la iniciaron antes de los 14 años de edad tuvieron 12.29 (IC 95\% 4.40-34.34) veces más posibilidad de tener antecedente de embarazo, de 13.84 (IC 95\% 6.55-29.13) veces cuando el inicio de relaciones sexuales sucedió entre los 14 y los 15 años; finalmente, haber iniciado relaciones sexuales a los 16 y 17 años tuvo 5.9 veces más posibilidad de antecedente de embarazo, en relación con aquellas mujeres que tuvieron su primera relación entre los 18 y los 19 años de edad.

\section{Discusión}

En este sector de la población, aún existe una brecha en cuanto al conocimiento de métodos anticonceptivos; los resultados de la ENSA 2000 muestran que sólo $54.2 \%$ de los adolescentes de entre 12 a 14 años de edad mencionaron conocer al menos un método anticonceptivo, en los adolescentes de 15 a 19 años de edad el porcentaje de conocimiento se incrementó a $78.8 \%$. Es difícil comparar los cambios en las proporciones del conocimiento de métodos anticonceptivos entre los y las adolescentes debido a la variabilidad en las edades consideradas por cada encuesta; además, la mayoría de las encuestas demográficas en cuestión de fecundidad se dirigen únicamente a la población femenina. Sin embargo, el porcentaje de conocimiento de métodos anticonceptivos es menor en la ENSA 2000 que el reportado por otras encuestas que indican que más de $80 \%$ de los adolescentes conocía al menos un método anticonceptivo. ${ }^{11,12}$ Otras estimaciones a mediados de la década de los ochenta han demostrado un incremento en el conocimiento de métodos anticonceptivos en las mujeres de 15 a 19 años de edad, pasando de $89.8 \%$ en 1987 a $93.4 \%$ en $1997,{ }^{10}$ mientras que en los resultados de la ENSA 2000 el porcentaje de conocimiento fue de $79.6 \%$ en este mismo grupo de la población.

La información analizada en este estudio indica que los adolescentes que necesitan de mayor información de métodos anticonceptivos son los que tienen menor edad, menor escolaridad, los que viven en áreas rurales, con menor ingreso y aquellos que no tienen acceso a servicios médicos.

En cuanto al patrón de inicio de la vida sexual en los y las adolescentes mexicanos los resultados de la ENSA 2000 muestran que $16.4 \%$ ha tenido relaciones 
sexuales; adicionalmente, se observa un incremento sostenido en la proporción de actividad sexual en los jóvenes de 12 a 17 años de edad de uno y otro sexo, sin embargo en el grupo de 18 a 19 años de edad el porcentaje de actividad sexual es mayor en los hombres que en las mujeres $(p=0.0000)$. Por otro lado, el promedio de la edad en la primera relación no sigue el mismo comportamiento observado en las encuestas previas que indican que los hombres inician su actividad sexual a edades más tempranas que las mujeres: los resultados de la ENSA 2000 muestran que la edad promedio de la primera relación sexual fue a los 15.7 años; no se observa diferencia significativa entre los hombres y las mujeres (15.7 años y 15.8 años, respectivamente, $p=0.0983$ ). Ahora bien, los resultados señalaron que el porcentaje de adolescentes que han iniciado su vida sexual es mayor en aquellos con menor nivel de escolaridad, los que no tienen acceso a servicios médicos y los que viven en poblaciones urbanas.

Los resultados de la ECRAMM, en 1988, destacan que sólo $33.8 \%$ de los jóvenes con actividad sexual habían utilizado algún método anticonceptivo en la primera relación sexual, ${ }_{1}^{13}$ y los resultados de la ENSA 2000 indicaron un incremento de tres puntos porcentuales en el uso de métodos en la primera relación sexual. Entre los métodos anticonceptivos usados por los adolescentes, el condón se reporta con mayor frecuencia $(87.7 \%)$, y le siguen en importancia los hormonales orales $(6.9 \%)$, el coito interrumpido (3.4\%), y el método del ritmo (2.5\%). Por otra parte, este estudio permite caracterizar al adolescente capaz de utilizar algún método anticonceptivo en su primera relación sexual: ser adolescente hombre, que cuenta con alta escolaridad, posee información sobre anticoncepción e inicia su vida sexual a una mayor edad.

Otro hallazgo de importancia fue que $55.7 \%$ de las adolescentes con actividad sexual dijeron haberse embarazado al menos una vez; se observó una tendencia en las estimaciones de antecedente de embarazo de acuerdo con la edad siguiendo el mismo patrón en las diferentes variables estudiadas. En este estudio se confirmó la asociación estadística entre el antecedente de embarazo y baja escolaridad que han descrito otros autores. ${ }^{21-23}$ Del mismo modo se identificó que el embarazo en la adolescencia está estrechamente relacionado con el inicio de actividad sexual antes de los 16 años de edad y con el estado civil: hallazgos similares han sido notificados en otro estudio. ${ }^{19}$

En resumen, la brecha existente entre el conocimiento y el uso de métodos anticonceptivos en la primera relación sexual es muy significativa y, de acuerdo con las condiciones de mayor riesgo, debe tomarse en cuenta en el momento de diseñar programas de salud reproductiva dirigidos a esta población que inicia su vida sexual.

Evaluaciones de programas de salud reproductiva proponen que la educación sexual es el medio idóneo para ayudar a jóvenes a prevenir esos problemas y mejorar su futura salud reproductiva. ${ }^{23}$ Dado que algunos adolescentes inician su vida sexual a los 12 años de edad la OMS recomienda que los programas estructurados de educación sexual empiecen mucho antes y plantea la creación de dos tipos de programas de educación sexual: los dirigidos a los adolescentes que no han tenido relaciones sexuales, y los diseñados para aquellos que ya las han tenido. ${ }^{24}$

Los resultados de este estudio permiten identificar el comportamiento sexual en los adolescentes del país; tenemos la idea de cómo se relacionan algunas variables con dicho comportamiento: la edad, el ingreso familiar, la urbanización y la escolaridad, aunque son controvertidos pues en algunos aspectos coinciden con los reportados, pero en otros parecen opuestos. Habrá que considerar que la población de adolescentes necesita mayor difusión de la educación sexual, así como reforzar las estrategias ya empleadas. Finalmente, los investigadores del comportamiento sexual en los adolescentes tienen una tarea inmensa; es necesario dedicar esfuerzos al desarrollo de metodologías uniformes y estandarizadas que permitan abarcar las complejidades de la sexualidad en su dimensión subjetiva, cultural y social.

\section{Agradecimientos}

Agradecemos a la actuaria Ivett Selene Quintana Nogueda por su apoyo en el procesamiento de los datos, y a los revisores de este documento por sus críticas y sugerencias, las cuales fueron de gran valor para la presentación final.

\section{Referencias}

1. O rganización Panamericana de la Salud. Salud de los adolescentes. W ashington, DC: O PS/O MS; 1995.

2. Magdaleno M, Munist MM, Serrano CV, Silver TJ. Sexualidad y adolescencia. La personalidad en el contexto de la salud integral de los adolescentes. En: La salud del adolescente y el joven.W ashington, DC: O rganización Panamericana de la Salud-O rganización Mundial de la Salud; 1994:1-17. Publicación Científica N úm. 6.

3. Monroy AL, Velasco L. Fecundidad en la adolescencia. Causas, riesgo y opciones. W ashington, DC:0 PS; 1988.

4. Pick S, Givaudan M, Saldívar-Garduño A. La importancia de los factores psicosociales en la educación sexual de los adolescentes. Perinatol Reprod Hum 1994;2:143-150. 
5. O rganización Panamericana de Salud. Plan de acción de desarrollo y salud de adolescentes y jóvenes en las A méricas, 1998-2001.

W ashington, D C:0 PS/O MS; 1998.

6. Santos-Preciado JL,Villa-Barragán JP, G arcía-Avilés MA, León-Alvarez GL, Q uezada-Bolaños S,Tapia-C onyer R. La transición epidemiológica de las y los adolescentes en México. Salud Publica Mex 2003;45 supl 1:S140-S152.

7. Cates W Jr, Rauh JL.A dolescents and sexually transmitted diseases:An expanding problem. Introduction. J Adolesc Health C are 1985;6(4): 257261.

8. Biro FM, Rosenthal SL.Adolescents and sexually transmitedd diseases: Diagnosis, developmental issues, and prevention.J Pediatr Health Care $1995 ; 9(6): 256-262$

9. Bunnell RE, Dahlberg L, Rolfs R, Ransom R, Gershman K, Farshy $C$ et al. High prevalence and incidence of sexually transmitted diseases in urban adolescent females despite moderate risk behaviors.J Infect D is 1999;180(5):1624-1631.

10. Consejo N acional de Población. Cuadernos de Salud Reproductiva. México, DF: Conapo; 2000.

11. Fundación Mexicana para la Planeación Familiar,AC. Encuesta para el programa Gente Joven 1999. México, DF:Mexfam; 1999.

12. Encuesta $N$ acional de la Juventud 2000. Resultados $G$ enerales. México, DF: Secretaría de Educación Pública, Instituto Mexicano de la Juventud; 2002.

13. García- Baltazar J, Figueroa-Perea JG, Reyes-Zapata H, Brindis C, Pérez-Palacios G. Características reproductivas de adolescentes y jóvenes en la Ciudad de México. Salud Publica Mex 1993;35:682-691. 14. Stern C. El embarazo en la adolescencia como problema público: una visión crítica. Salud Publica Mex 1997;39:137-143.

15. Consejo Nacional de Población 2000. La situación actual de las y los jóvenes en México. Diagnóstico demográfico. México, DF: Conapo; 1997:16
16. Escobedo E, Fletes J,Velásquez V. Embarazo en adolescentes: seguimiento de sus hijos durante el primer año de vida. Bol Med Hosp Infant Mex 1995;52(7):415-419.

17. Casanueva E, Soberanis Y, O rtiz T, Bobadilla MA. Cambios en la composición corporal en el periodo perinatal en un grupo de adolescentes. Perinatol Reprod Hum 1991;5(1):28-32.

18. Schlaepfer L, Infante C. Patrones de inicio de la vida reproductiva: su relación con la mortalidad infantil y comportamientos reproductivos futuros. En: Lartigue T, Avila $\mathrm{H}$, ed. Sexualidad y reproducción humana en México. México, DF: UIA-Plaza y Valdez; 1996;2:77-94.

19. Menkes C, Suárez L. Sexualidad y embarazo de adolescentes en México. México, DF: Universidad N acional Autónoma de México; 2003: 233-263. Papeles de Población N 0. 35.

20.Valdespino JL, 0 laiz G, López-Barajas MP, Mendoza L, Palma O, Velásquez 0 et al. Encuesta N acional de Salud 2000.Tomo I.Vivienda, población y utilización de servicios. Cuernavaca, Morelos, México. Instituto N acional de Salud Pública; 2003.

21. Pick de W eiss S,Atkin LC, G ribble JN , Andrade-Palos P. Sex, contraception, and pregnancy among adolescents in Mexico City. Stud Fam Plann 1991;22:74-82.

22. Munist M, Giurgiovich A, Solís JS, Mora GE. Factores relacionados con la fecundidad en la adolescencia. La salud del adolescente y del joven. W ashington, DC: O PS/O MS; 1995 Publicación Cientifica N 0. 522. 23. Pick de W eiss S,Andrade-Palos P,Townsend J, G ivaudan M. Evaluación de un programa de educación sexual sobre conocimientos, conducta sexual y anticoncepción en adolescentes. Salud Ment 1994;17:25-35.

24. G runseit A, Kippax S. Effects of sex education on young people's sexual behavior. Ginebra: O MS; 1993:5,6. 